\title{
THE RADIOCARBON CONTENT OF INDIVIDUAL LIGNIN-DERIVED PHENOLS: TECHNIQUE AND INITIAL RESULTS
}

\author{
A P McNichol ${ }^{1,2} \cdot \mathrm{J} \mathrm{R}$ Ertel $^{3} \bullet$ T I Eglinton ${ }^{1}$
}

\begin{abstract}
We present a method for the isolation of phenolic compounds derived from lignin for radiocarbon analysis. These phenols are generated by chemical oxidation of polymeric materials and derivatized for separation and recovery by preparative capillary gas chromatography (PCGC). This technique yields tens of micrograms of pure, stable compounds that can be converted to graphite and analyzed by accelerator mass spectrometry (AMS). Analysis of model flavor compounds and dated woods indicates that, in most cases, the radiocarbon $\left({ }^{14} \mathrm{C}\right)$ contents of the individual compounds, corrected for the contribution of the derivative, agree with that of the bulk material to within $20 \%$.
\end{abstract}

\section{INTRODUCTION}

Understanding the fate of terrestrial organic matter is critical to understanding the global carbon cycle and its response to anthropogenic perturbations. Organic matter synthesized in the terrestrial biosphere can undergo a number of different fates, e.g. storage in soils, transport to estuaries and oceans via run-off, and oxidation to $\mathrm{CO}_{2}$, on both short and long time scales. Chemical and isotopic tracer studies of biomarker compounds, individual molecules that are unique to a particular source or process, have all proven useful tools for deciphering this cycle, yet several aspects of this cycle remain poorly constrained. By isolating and dating biomarker compounds unique to the terrestrial environment in different downstream organic carbon reservoirs, e.g. soils, sediments, and riverine and oceanic dissolved organic carbon (DOC), we can determine the reactivity and residence time of terrestrial organic matter in different global carbon pools. To accomplish this, it is necessary to isolate individual source-specific biomarkers from the complex organic matrix found in the environment. Preparative capillary gas chromatography (PCGC) coupled to micro-scale accelerator mass spectrometric (AMS) measurements of ${ }^{14} \mathrm{C}$ content has been used to date individual lipid-like compounds that have been extracted from natural samples including hydrocarbons, fatty acids, and sterols (Eglinton et al. 1996). In this paper, we extend this approach to macromolecular lignin components that represent a major portion of the global carbon pool, but that require chemical oxidation and derivatization prior to analysis.

Lignins are biopolymers that are unique to vascular plants (Sarkanen and Ludwig 1971) and encompass up to $30 \%$ of the carbon in woody components. Upon decay of vascular plant material in soils, lignins become part of the macromolecular soil organic matter (SOM) pool and are leached into rivers as dissolved humic substances. Additionally, lignins may be removed from their original site of deposition by the physical transport of material such as leaves and wood pieces. Lignins can be detected chemically as a set of unambiguous "lignin-derived" phenols that are released by chemical oxidation and analyzed, after derivatization, by gas chromatography. The chemical structures of some important lignin phenols are shown in Figure 1. Lignin-derived phenols are produced from lignin biopolymers as "families" of compounds of similar methoxyl content but different oxidation states. The relative abundance of the families of phenols provides information about the types of vascular plants from which the material was derived. Hedges and Mann (1979) showed that the relative abundance of vanillyl, syringyl, and cinnamyl phenols can be used to characterize material originating from gymnosperms (non-flowering plants including conifers), angiosperms (flowering plants including hardwood trees, herbs, and grasses), or the non-wood vascular plant tissue (leaves

\footnotetext{
${ }^{1}$ Woods Hole Oceanographic Institution, Woods Hole, Massachusetts 02543, USA

${ }^{2}$ Email: amcnichol@whoi.edu

${ }^{3}$ Lawrence Livermore National Laboratory, Livermore, California 94550, USA
} 
and needles). Coupling this information with the age of the different component will provide a powerful tracer of terrestrial carbon in the environment.

The additional chemical treatments required for the analysis of lignin-derived phenols increases the possibility of contamination and isotopic fractionation. In this paper, we evaluate the effect of chemical oxidation and derivatization on the isotopic composition of individual compounds isolated by PCGC by using model flavor compounds related to lignin-derived phenols and woods of known ages. The ability of the lignin oxidation technique to generate families of highly related compounds of different oxidation states allows for a definitive test of the entire procedure.

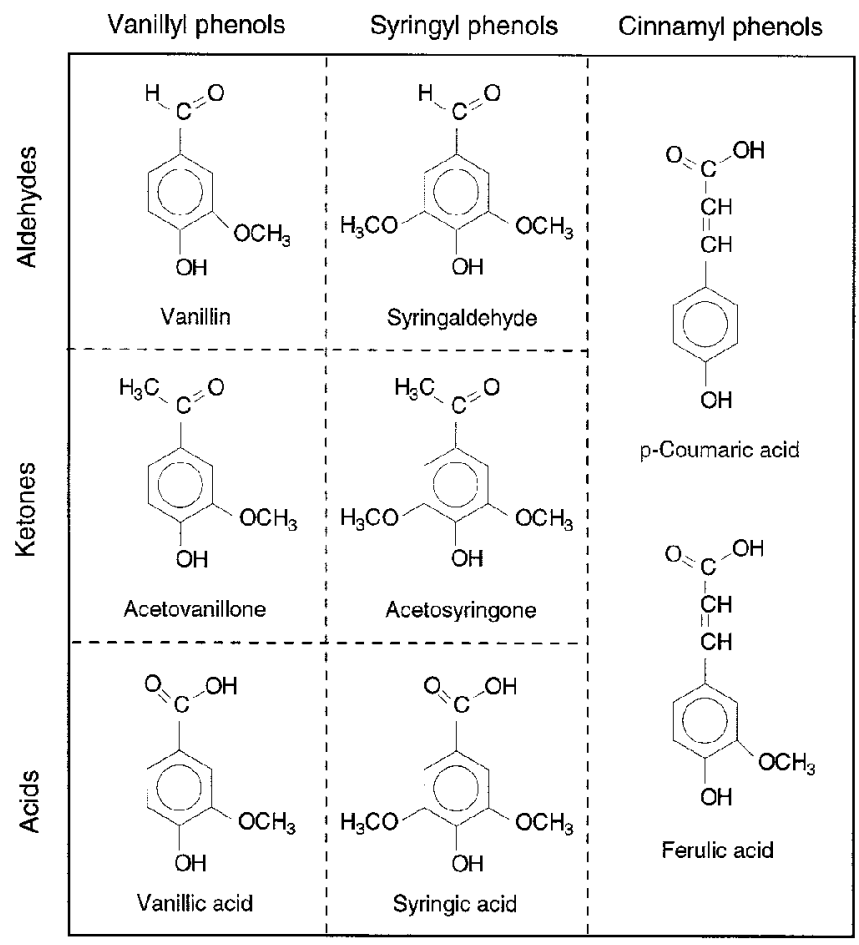

Figure 1 Lignin-derived phenols isolated and analyzed by $\mathrm{CuO}$ oxidation

\section{METHODS}

For this study, we analyzed the ${ }^{14} \mathrm{C}$ content of organic compounds obtained from two sources-commercially available flavor compounds and oxidative hydrolysis products of wood samples. We purchased vanillin and cinnamic acid standards that were both isolated from natural plant material and produced synthetically, presumably from petrochemical materials (natural, Aldrich Chemical Company Lot \#03803DQ, and synthetic, Supelco Lot \#LA-52245, vanillin; natural, Supelco Lot \#LA53695, and synthetic, Supelco Lot \#LA-51290, cinnamic acid). The reported isotope activities for the natural compounds are $15.20 \pm 0.38 \mathrm{dpm} / \mathrm{gC}(\mathrm{Fm}=1.1213)$ for vanillin and $15.49 \pm 0.45 \mathrm{dpm} / \mathrm{gC}(\mathrm{Fm}$ $=1.1427$ ) for cinnamic acid. Additionally, a $\delta^{13} \mathrm{C}$ value of $-31.01 \pm 0.13 \%$ is reported for natural vanillin. We prepared standard solutions of the two natural compounds, the two synthetic compounds and a 1:1 mixture of these solutions and separated all three solutions by PCGC for ${ }^{14} \mathrm{C}$ measurements. We obtained three wood samples, chosen to represent a range of different radiocarbon concentrations, 
and dated the bulk wood samples. Two Creeks Wood, a subfossil wood from Wisconsin, USA, was one of the samples distributed as part of the IAEA ${ }^{14} \mathrm{C}$ Intercomparison Exercise 1990 (Rozanski et al. 1992); the consensus fraction modern value for ${ }^{14} \mathrm{C}$ is $0.2305 \pm 0.02$ and for $\delta^{13} \mathrm{C}$, the consensus value is $-25.49 \pm 0.72 \%$ o. We anticipated that juniper wood collected from King Midas' Tomb, Turkey (Nelson et al. 1995) would have an age around that of the tomb, 2700 BP $(\mathrm{Fm}=0.7290)$. Finally, we analyzed a modern oak wood, anticipating an age close to present (Fm approx. $=1.000)$.

Phenolic compounds were released from the lignin component of the woods using oxidative hydrolysis with alkaline $\mathrm{CuO}$ according to the technique of Hedges and Ertel (1982). However, with these samples the hydrolysis products were derivatized with dimethyl sulfate prior to chromatography using the procedure summarized below (Vogel 1996). The reaction adds a methyl group to each free hydrogen on a hydroxyl or carboxyl group and converts the phenols and acids to compounds amenable to gas chromatographic (GC) analysis. Standards or $\mathrm{CuO}$ oxidation products were transferred to test tubes in dry acetone and evaporated to dryness under a stream of $\mathrm{N}_{2}$. Dimethyl sulfate in an approximately two-fold excess, $10-20 \mathrm{mg} \mathrm{K} \mathrm{CO}_{3}$, and approximately $2 \mathrm{~mL}$ of dry acetone were added and stirred at $70{ }^{\circ} \mathrm{C}$ overnight. The excess dimethyl sulfate was destroyed with a few drops of $30 \%$ ammonium hydroxide solution by stirring for one hour. (Note: Dimethyl sulfate is a potent carcinogen.) The derivatized phenols were extracted with ether, dried with sodium sulfate, and prepared for chromatography. We have found the efficiency of dimethyl sulfate derivatization is $>90 \%$ in most instances and appears to be related to the complexity of the $\mathrm{CuO}$ mixtures. Individual phenols were isolated in sufficient quantity for AMS analysis from the hydrolyis products by PCGC. The chromatographic conditions were similar to those reported in Eglinton et al. (1996) except that a 60-m DB-5 fused silica column was used for separating the methylated derivatives. For these phenol-rich samples, as few as 36 repeated injections were required to collect enough material. For many geochemical or environmental samples, more than 100 injections may be required. A typical chromatogram with the peaks isolated in this study identified is shown in Figure 2. After isolation of the individual phenols, each sample was transferred to a Vycor combustion tube with solvent. A small aliquot was removed for analysis by GC, GC-mass spectrometry (GC-MS), and/or isotope ratio monitoring-GC-MS (irm-GC-MS) to check its yield, purity, identification, and stable carbon isotopic composition; the remainder was evaporated to dryness in a stream of $\mathrm{N}_{2}$.

We also measured the ${ }^{14} \mathrm{C}$ content of the flavor compounds prior to GC analysis, the bulk wood samples, and the derivatizing agent in order to use a mass balance to calculate the fraction modern of the original phenol. Standard combustion and graphite preparation techniques were used (McNichol et al. 1994).

In order to assess whether the measurement of a very small sample isolated from the PCGC on the accelerator creates particular problems, we treated the vanillin samples isolated from two of the wood samples in the following manner. We collected enough sample in the PCGC trap to prepare a "routine" graphite sample, i.e. containing more than $500 \mu \mathrm{g}$ carbon. We then split the sample into two fractions, one containing enough carbon for a small sample (approx. $100 \mu \mathrm{g} \mathrm{C}$ ) and one with enough for a routine sample. Graphite targets were made from both fractions.

After evaporation of the solvent, $100 \mathrm{mg}$ of $\mathrm{CuO}$ was added to the combustion tube, the tube was evacuated for approximately $45 \mathrm{sec}$ to a pressure of $<2$ mTorr while immersed in dry-ice/isopropanol bath, and the tube was flame-sealed. Samples were combusted at $850{ }^{\circ} \mathrm{C}$ for $5 \mathrm{hr}$; the resultant $\mathrm{CO}_{2}$ was purified, quantified, and converted to graphite (McNichol et al. 1995). A small portion, usually $<10 \%$ of the sample, was split and transferred to a VG Prism for stable isotope analysis. Samples containing less than $200 \mu \mathrm{g}$ carbon were treated as "small samples" and analyzed accord- 
ing to special procedures (Pearson et al. 1998) and all other samples were analyzed according to our standard procedures (McNichol et al. 1994). ${ }^{14} \mathrm{C}$ values are reported as fraction modern and $\Delta{ }^{14} \mathrm{C}$ as described in Stuiver and Polach (1977), Stuiver (1980), and Donahue et al. (1990). We report the error determined during AMS analysis as described in Schneider et al. (1995).

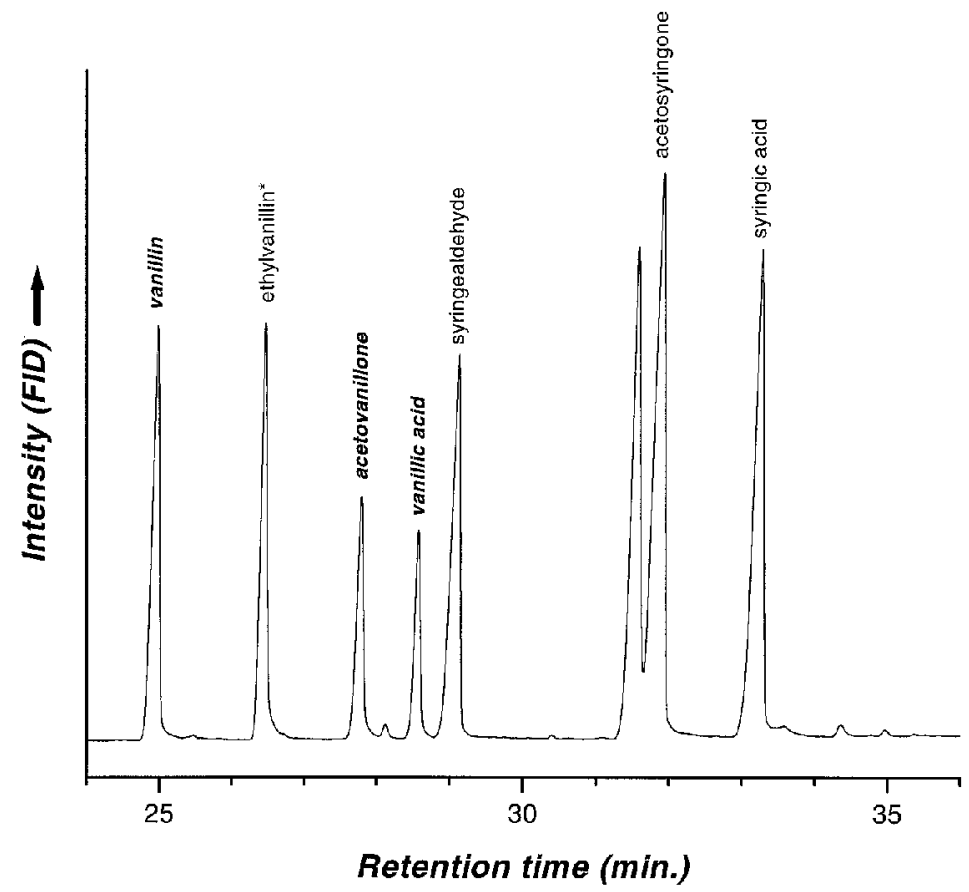

Figure 2 Representative chromatogram identifying the peaks isolated in this study

\section{RESULTS}

We chose to analyze model flavor compounds and lignin-derived phenols isolated from wood to test different aspects of our procedure. The flavor compounds allowed us to test the derivatization and chromatographic methods while the wood samples allowed us to test the entire chemolysis, derivatization and chromatographic procedure. The compounds we analyzed are shown in their underivatized state in Figure 1; after derivatization, the free $\mathrm{H}$ on any hydroxyl or carboxyl group is replaced with a methyl group. Although we isolated six lignin-derived phenols from each wood sample, for this study we chose only two phenols, both vanillyl, on which to measure ${ }^{14} \mathrm{C}$. There is no reason syringyl and cinnamyl phenols cannot be measured with this method and we have successfully done so in other studies. To calculate the ${ }^{14} \mathrm{C}$ content of the underivatized compound, we use the mass balance

$$
\mathrm{R}_{\mathrm{cpd}}=\left(\mathrm{R}_{\mathrm{t}} \mathrm{C}_{\mathrm{t}}-\mathrm{R}_{\mathrm{d}} \mathrm{C}_{\mathrm{d}}\right) / \mathrm{C}_{\mathrm{cpd}}
$$

where

$\mathrm{R}$ is the isotope ratio $\left(\delta^{13} \mathrm{C}, \Delta^{14} \mathrm{C}\right)$ of the compound (cpd), derivative (d), and derivatized compound (t), and

$\mathrm{C}$ is the amount of carbon. 
The GC analysis of phenols has traditionally been accomplished by derivatizing the exchangeable hydrogens with bis-trimethylsilylfluoroacetamide (BSTFA) producing trimethylsilyl (TMS) derivatives. The use of TMS derivatives of lignin phenols is rapid, quantitative, and free of isotopic fractionation (Goni and Eglinton 1996), but the TMS derivatives are unstable and add three to six carbon atoms per molecule. Partial loss of the TMS derivatives after PCGC separation would be undetectable, but could dramatically affect the ${ }^{14} \mathrm{C}$ content. Initial attempts to use this procedure resulted in unacceptable breakdown of the derivatives during PCGC separation, so the more stable methyl derivatives were synthesized.

\section{Isotopic Values of Derivative Carbon}

The fraction modern of the derivative we measured and use in the calculations was 0.0018 . This is essentially a background level, indicating the compound has virtually no ${ }^{14} \mathrm{C}$ in it and is most likely synthesized from petroleum products. We measured a $\delta^{13} \mathrm{C}$ of -51.37 for dimethyl sulfate, the derivatizing agent, but we have found that it is more appropriate to use a value of $-100 \%$ o for the $\delta^{13} \mathrm{C}$ of $\mathrm{C}_{\mathrm{d}}$. The analysis of the methylated lignin phenol derivatives by irm-GC-MS suggests there is a significant fractionation during the derivatization reaction (Ertel and Freeman, unpublished data). Based on results from another study, we believe it is reasonable to assume that any fractionation observed occurs during derivatization, not isolation, and that the magnitude of isotopic fractionation is similar for each of the individual lignin phenols. Goni and Eglinton (1996) demonstrated that there was no significant isotopic fractionation associated with the liberation of individual lignin phenols from wood by $\mathrm{CuO}$ oxidation. Additionally, they showed that the $\delta^{13} \mathrm{C}$ of TMS derivatized to 13 lignin-derived phenols was the same within $1 \%$. Because of the lack of ${ }^{14} \mathrm{C}$ in the derivatizing agent, isotopic fractionation of the type inferred for ${ }^{13} \mathrm{C}$ has no influence on the calculation of ${ }^{14} \mathrm{C}$ for the derivatized compound.

The stable isotope data (Figure 3, Table 1) for the flavors and woods indicate that there is no significant isotopic fractionation introduced during isolation, derivatization, and chromatography. For vanillin, our measured $\delta^{13} \mathrm{C}(-31.21 \pm 0.05)$ agrees well with the reported value $(-31.01 \pm 0.13)$. The values measured on the compounds after chromatography, both flavor and wood, agree with those measured on the bulk sample within $2 \%$. Although this range was somewhat larger than we had hoped, it appears to be random and it is not large enough to affect our $\Delta^{14} \mathrm{C}$ values. We believe that some of the scatter is caused by a variable fractionation during derivatization, possibly due to competitive reactions. In this case, using a value of $-100 \%$ o for $\mathrm{C}_{\mathrm{d}}$ may only be appropriate when certain conditions are met, e.g. ensuring there is an excess of derivatizing agent. Although it is not crucial to be able to make accurate stable isotope measurements with this method, valuable geochemical information is lost in its absence. Source identification and biochemical pathway information, e.g. C3 vs. C4 plants, are lost if we cannot make an accurate measurement. It will be possible to make these measurements using irm-GC-MS, but this is an expensive alternative which requires additional material.

The ${ }^{14} \mathrm{C}$ data (Figure 4, Table 1) indicate that we are able to accurately reproduce the original $\Delta^{14} \mathrm{C}$ for samples containing between 50-500 $\mu \mathrm{g}$ carbon. The values we measured for the bulk natural flavor compounds by AMS agree with the reported values to within $6 \%$. For both the flavor and wood samples, the values measured after chromatography agree with those measured on the bulk sample to within $20 \%$, for all but two compounds, and the results display no systematic offsets. Moreover, the flavor and wood-derived compounds behaved equally well which suggests that the $\mathrm{CuO}$ oxidation used to liberate the lignin-derived phenols does not contribute significantly to the error in these measurements. Comparison of the ${ }^{14} \mathrm{C}$ results from the two vanillin samples isolated from the Two Creeks and Juniper woods indicate that the size of the sample analyzed after chromatography does not affect 
the results. Comparing vanillic acid and cinnamic acid to vanillin shows that the number of hydroxyl groups derivatized does not affect the calculated results, although it is likely that there is a loss of precision with a higher ratio of derivative carbon to total carbon (Goni and Eglinton 1996). We believe that as we gain experience with this technique, the precision will ultimately be limited by the ability to measure small samples on the accelerator.

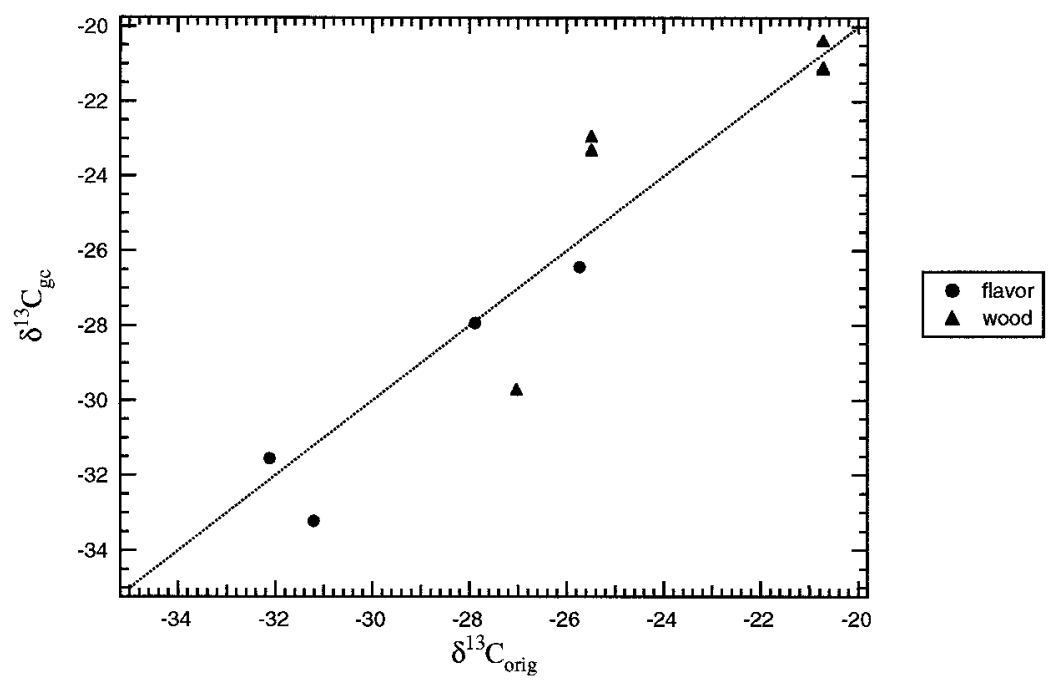

Figure 3 Values of $\delta^{13} \mathrm{C}$ measured on flavor and lignin-derived phenols isolated by GC after correction for derivative carbon relative to the $\delta^{13} \mathrm{C}$ of the original material. The line shown is the expected $1: 1$ ratio.

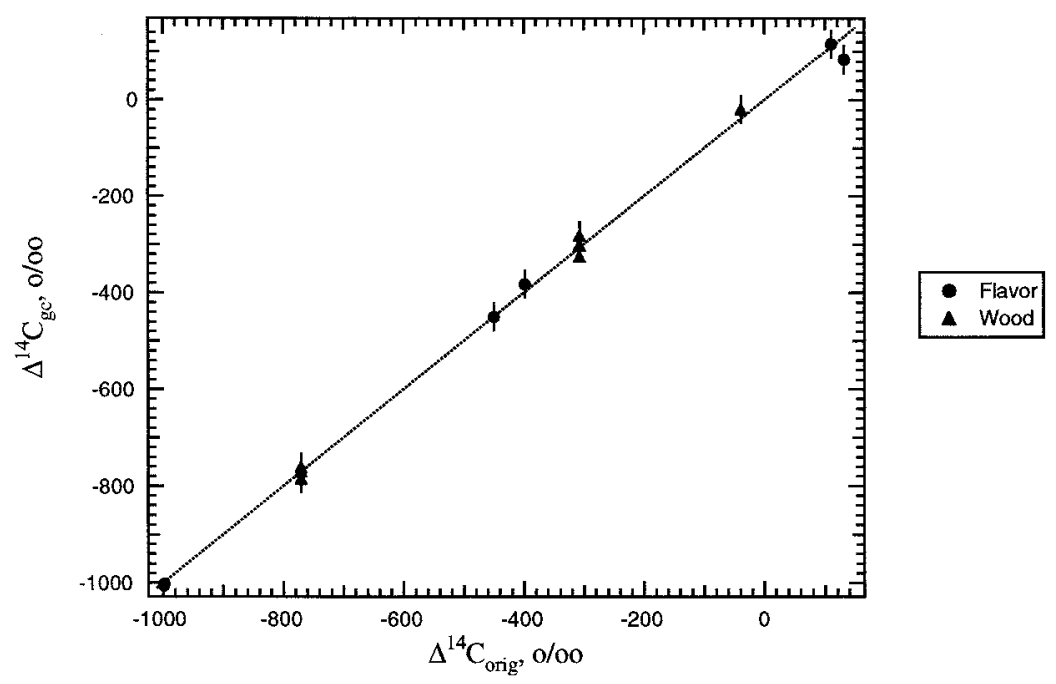

Figure 4 Values of $\Delta^{14} \mathrm{C}$ measured on flavor and lignin-derived phenols isolated by GC after correction for derivative carbon relative to the $\Delta^{14} \mathrm{C}$ of the original material. The line shown is the expected $1: 1$ ratio. 
Table 1 Stable and ${ }^{14} \mathrm{C}$ isotopic values for flavor and wood samples reported as $\delta^{13} \mathrm{C}$ (\%o relative to VPDB), fraction modern (Fm), and $\Delta^{14} \mathrm{C}(\% \circ)^{\mathrm{a}}$

\begin{tabular}{|c|c|c|c|c|c|c|c|c|}
\hline \multirow[b]{2}{*}{ Flavors } & \multicolumn{2}{|c|}{$\# \mathrm{C}$} & \multirow[b]{2}{*}{$\delta^{13} \mathrm{C}$} & \multirow[b]{2}{*}{$\mathrm{Fm}$} & \multirow[b]{2}{*}{ err } & \multirow[b]{2}{*}{$\Delta^{14} \mathrm{C}$} & \multirow[b]{2}{*}{ err } & \multirow[b]{2}{*}{$\Delta(\Delta)$} \\
\hline & $\mathrm{C}$ & $\mathrm{D}$ & & & & & & \\
\hline \multicolumn{9}{|l|}{ Synthetics } \\
\hline Cinnamic acid & & & & 0.0017 & 0.0008 & -998.3 & 0.8 & \\
\hline Cinnamic acid, PCGC & 9 & 1 & -29.31 & -0.0020 & 0.0014 & -1001.6 & 1.4 & -3.3 \\
\hline Vanillin & & & & 0.0009 & 0.0008 & -999.1 & 0.8 & \\
\hline Vanillin, PCGC & 8 & 1 & -28.98 & -0.0060 & 0.0013 & -1005.9 & 1.3 & -6.8 \\
\hline \multicolumn{9}{|l|}{ Natural } \\
\hline Cinnamic acid & & & -25.73 & 1.1370 & 0.0093 & 131.3 & 9.3 & \\
\hline Cinnamic acid, PCGC & 9 & 1 & -26.44 & 1.0897 & 0.0098 & 84.3 & 9.8 & -47.1 \\
\hline Vanillin & & & -31.21 & 1.1154 & 0.0069 & 109.8 & 6.9 & \\
\hline Vanillin, PCGC & 8 & 1 & -33.22 & 1.1352 & 0.0100 & 129.5 & 10.0 & 19.7 \\
\hline \multicolumn{9}{|l|}{ 1:1 Mix } \\
\hline Cinnamic acid, expected & & & -27.89 & 0.5529 & & -449.9 & & \\
\hline Cinnamic acid & 9 & 1 & -27.93 & 0.5523 & 0.0062 & -450.5 & 6.2 & -0.6 \\
\hline Vanillin, expected & & & -32.12 & 0.6037 & & -399.3 & & \\
\hline Vanillin & 8 & 1 & -31.55 & 0.6208 & 0.0088 & -382.3 & 8.8 & 17.0 \\
\hline \multicolumn{9}{|l|}{ Woods } \\
\hline \multicolumn{9}{|l|}{ Juniper } \\
\hline Bulk Wood & & & -20.73 & 0.6959 & 0.0028 & -307.6 & 2.8 & \\
\hline Vanillin, large & 8 & 1 & -21.13 & 0.6787 & 0.0065 & -324.7 & 6.5 & -17.1 \\
\hline Vanillin, small & 8 & 1 & -21.09 & 0.7214 & 0.0167 & -282.2 & 16.7 & 25.4 \\
\hline Vanillic acid & 8 & 2 & -20.37 & 0.7017 & 0.0094 & -301.8 & 9.4 & 5.7 \\
\hline \multicolumn{9}{|l|}{ Two Creeks Wood } \\
\hline Bulk Wood & & & -25.49 & 0.2305 & & -770.6 & & \\
\hline Vanillin, large & 8 & 1 & -23.30 & 0.2317 & 0.0027 & -769.5 & 2.7 & 1.1 \\
\hline Vanillin, small & 8 & 1 & -22.92 & 0.2166 & 0.0081 & -784.5 & 8.1 & -13.9 \\
\hline Acetovanillon & 9 & 1 & -23.29 & 0.2400 & 0.0077 & -761.2 & 7.7 & 9.4 \\
\hline \multicolumn{9}{|l|}{ Modern White Oak Wood } \\
\hline Bulk Wood & & & -27.03 & 0.9661 & 0.0052 & -38.7 & 5.2 & \\
\hline Vanillin & 8 & 1 & -29.71 & 0.9855 & 0.0109 & -19.4 & 10.9 & 19.3 \\
\hline
\end{tabular}

${ }^{a} \mathrm{All} \delta^{13} \mathrm{C}$ and ${ }^{14} \mathrm{C}$ values were measured or, for the samples isolated by PCGC, measured and corrected for derivative carbon except in the following cases. The expected values for the 1:1 mix were calculated using the isotope ratios measured on the synthetic and natural compounds and the amounts of each used to prepare the mixture. The \#C lists the number of carbon atoms in the original molecule (C) and the number of carbons added during derivatization (D).

\section{DISCUSSION}

The combination of techniques to isolate lignin-derived phenols from complex matrices with preparative capillary gas chromatography should provide an exciting tool for understanding the cycling of terrestrial carbon in the global environment. The extension of the technique to natural samples is likely to present some challenges. The compounds isolated in this study were generated from either pure compounds or isolated from a relatively pure substrate-wood—and generated "clean" chromatograms in which many of the compounds of interest were completely resolved from one another. 
Based on our experience with other compounds and compound classes isolated from geochemical samples, a main criterion determining the quality of ${ }^{14} \mathrm{C}$ data is the chromatographic separation of the compounds of interest (Eglinton et al. 1996). Isolation methods applied to soils, sediments or other natural matrices will yield more complex mixtures that may require additional steps to produce cleaner fractions or the use of multi-dimensional GC separation techniques. The validity of the basic technique coupled with our successes with other compounds suggests that these challenges can be successfully overcome. Coupled with stable and ${ }^{14} \mathrm{C}$ analyses of other important biomarker compounds, we will be able to decipher more of the information contained in the bulk organic carbon pool.

The techniques developed in this study can be used to study issues other than carbon cycling. For example, the ability to measure ${ }^{14} \mathrm{C}$ in lignin-derived phenols provides an alternate method for the determination of ${ }^{14} \mathrm{C}$ in tree rings. Wood samples of a sufficient size $(10-50 \mathrm{mg})$ to generate enough sample for a precise ${ }^{14} \mathrm{C}$ analysis $(>300 \mu \mathrm{g} \mathrm{C}$ ) could be used to provide data as a check on cellulose studies as well as in areas where lignin is preserved better than cellulose, e.g. Benner et al. (1990).

The techniques described here may also be useful for determining the provenance of flavors, fragrances, and other compounds used in commercial products. Products using flavors, such as vanillin, derived from natural substances command a greater price on the market, in part due to their reduced availability and the greater expense incurred in isolating the flavor. Thus, there is a strong financial incentive to pass the more-cheaply produced synthetic flavors off as natural ones. Often, the synthetic compounds are made from petroleum-derived carbon and have no detectable ${ }^{14} \mathrm{C}$, while the natural flavors have ${ }^{14} \mathrm{C}$ concentrations reflecting that of the atmosphere. An industry has grown around developing the means to detect these so-called "fraudulent molecules" (Hoffman 1997) and the techniques described here may add one more tool to this industry.

\section{ACKNOWLEDGMENTS}

We would like to thank Joanne Donoghue, Bryan Benitez-Nelson, and the NOSAMS staff for technical assistance on this project. We thank Michaele Kashgarian and other members of the CAMS Facility for the flavor compound radiocarbon analyses. John Hedges provided the Juniper wood sample. This work was supported by the National Science Foundation through Cooperative Agreements NSF OCE-801015, OCE-807266 and grant OCE-815568, and the Department of Energy through grant DE-FG02-92ER61428. This is WHOI Contribution \#10179.

\section{REFERENCES}

Benner R, Weliky K, Hedges JI. 1990. Early diagenesis of mangrove leaves in a tropical estuary: molecularlevel analyses of neutral sugars and lignin-derived phenols. Geochimica et Cosmochimica Acta 54:19912000.

Donahue DJ, Linick TW, Jull AJT. 1990. Isotope-ratio and background corrections for accelerator mass spectrometry radiocarbon measurements. Radiocarbon 32(2):135-42.

Eglinton TI, Aluwihare LI, Bauer JE, Druffel ERM. 1996. Gas chromatographic isolation of individual compounds from complex matrices for radiocarbon dating. Analytical Chemistry 68:904-12.

Goñi MA, Eglinton TI. 1996. Stable carbon isotopic analyses of lignin-derived $\mathrm{CuO}$ oxidation products by isotope ratio monitoring-gas chromatography-mass

spectrometry (irm-GC-MS). Organic Geochemistry 24:601-15.

Hedges JI, Ertel JR. 1982. Characterization of lignin by gas capillary chromatography of cupric oxide oxidation products. Analytical Chemistry 54:174-8.

Hedges JI, Mann DC. 1979. The characterization of plant tissue by their lignin oxidation products. Geochimica et Cosmochimica Acta 43:1803-7.

Hoffmann R. 1997. Fraudulent Molecules. American Scientist 85:314-7.

McNichol AP, Gagnon AR, Osborne EA, Hutton DL, Von Reden KF, Schneider RJ. 1995. Improvements in procedural blanks at NOSAMS: reflections of improvements in sample preparation and accelerator operation. Radiocarbon 37(2):683-91.

McNichol AP, Osborne EA, Gagnon AR, Fry B, Jones 
GA. 1994. TIC, TOC, DIC, DOC, PIC, POC—unique aspects in the preparation of oceanographic samples for ${ }^{14} \mathrm{C}$-AMS. Nuclear Instruments and Methods in Physics Research B 92:162-5.

Nelson BC, Goni MA, Hedges JI, Blanchette RA. 1995. Soft-rot fungal degradation of lignin in 2700 year old archaeological woods. Holzforschung 49:1-10.

Pearson A, McNichol AP, Schneider RJ, Von Reden KF. 1998. Microscale AMS measurements at NOSAMS. Radiocarbon 40(1):61-75.

Rozanski K, Stichler W, Gonfiantini R, Scott EM, Beukens RP, Kromer B, Van der Plicht J. 1992. The IAEA ${ }^{14} \mathrm{C}$ intercomparison exercise 1990. Radiocarbon 34(3):506-19.
Sarkanen KV, Ludwig CH. 1971. Lignins. Wiley-Interscience.

Schneider RJ, McNichol AP, Nadeau MJ, Von Reden KF. 1995. Measurements of the Oxalic Acid II/Oxalic Acid I ratio as a quality control parameter at NOSAMS. Radiocarbon 37(2):693-6.

Stuiver M. 1983. International agreements and the use of the new oxalic acid standard. Radiocarbon 25(2):7935.

Stuiver M, Polach HA. 1977. Discussion: reporting of ${ }^{14} \mathrm{C}$ data. Radiocarbon 19(3):355-63.

Vogel AS. 1996. Vogel's textbook of practical organic chemistry. 6th edition. London: Longman. 